\title{
Preventing ischial pressure ulcers: III. Clinical pilot study of chronic neuromuscular electrical stimulation
}

\author{
Hilton M. Kaplan, ${ }^{\mathrm{a}, *}$, Lucinda L. Baker ${ }^{\mathrm{b}}$, Salah Rubayi ${ }^{\mathrm{c}}$ and Gerald E. Loeb ${ }^{\mathrm{a}}$ \\ ${ }^{a}$ Department of Biomedical Engineering and Alfred Mann Institute for Biomedical Engineering, \\ University of Southern California, Los Angeles, CA, USA \\ ${ }^{\mathrm{b}}$ Department of Biokinesiology and Physical Therapy, University of Southern California, Los Angeles, CA, USA \\ ${ }^{\mathrm{c}}$ Pressure Ulcer Management Program, Rancho Los Amigos National Rehabilitation Center, Downey, CA, USA
}

\begin{abstract}
Objective: $\quad \mathrm{BIONs}^{\mathrm{TM}}$ (BIOnic Neurons) are injectable, wireless microstimulators that make chronic BION Active Seating (BAS) possible for pressure ulcer prevention (PUP). Neuromuscular electrical stimulation (NMES) produces skeletal motion and activates trophic factors, counteracting three major etiological mechanisms leading to pressure ulcers (PUs): immobility, soft-tissue atrophy, and ischemia. Companion papers I and II reviewed prior experience with NMES for PUP, and analyzed the biomechanical considerations, respectively. This paper presents a treatment strategy derived from this analysis, and the clinical results of the first three cases.

Methods: Two BIONs implanted (one on inferior gluteal nerve to gluteus maximus (GM), and other on sciatic nerve to hamstrings (HS)), in 3 spinal cord injured (SCI) subjects already undergoing gluteal rotation flaps for PUs. BAS using HS when seated, and BION Conditioning (BC) via GM+HS when non-weightbearing. Follow-up: 1 yr, including 6 mo. treatment window (interface pressure mapping; muscle perfusion scans; MRI, X-ray volume assessments).

Results: Successfully implanted and activated both desired muscle groups, selectively, in all. No PU recurrences or wound complications. Two subjects completed protocol. Mean results: Interface: contact pressure $-10 \%$; maximum pressure $-20 \%$; peak pressure area $-15 \%$. Vascularity: $\mathrm{GM}+20 \%$, HS $+110 \%$. Perfusion: $\mathrm{GM}+70 \%$, $\mathrm{HS}+440 \%$. Muscle volume: $\mathrm{GM}+14 \%$, HS $+31 \%$. Buttock soft-tissue padding: $+49 \%$. 1 BION failed; 1 BION rotated under GM.

Conclusions: Promising proof-of-concept data supporting the feasibility of implanted microstimulators to achieve sufficiently strong and selective activation of target muscles for PUP. Ultimate goal is prophylactic deployment through bilateral, nonsurgical injection of BIONs in chronically immobile patients.
\end{abstract}

Keywords: BION ${ }^{\mathrm{TM}}$ microstimulator, BION active seating, BION conditioning, neuromuscular electrical stimulation, pressure ulcer prevention, gluteal rotation flap

\section{Abbreviations}

AMI

Alfred Mann Institute at the University of Southern California, Los Angeles, CA

*Corresponding author: Hilton M. Kaplan, MD, PhD, PO Box 2337, Beverly Hills, CA 90213, USA. Tel.: +1 (310) 570 2822; Fax: +1 (310) 274 9931; E-mail: hkaplan@alumni.usc.edu.

$\begin{array}{ll}\text { AP } & \text { Antero-Posterior } \\ \text { BIONTM } & \text { BIOnic Neuron } \\ \text { BAS } & \text { BION Active Seating } \\ \text { BC } & \text { BION Conditioning } \\ \text { DC } & \text { Discharge } \\ \text { EM } & \text { Electromagnetic } \\ \text { GM } & \text { Gluteus Maximus } \\ \text { HS } & \text { Hamstrings }\end{array}$

1176-2322/11/\$27.50 @ 2011 - IOS Press and the authors. All rights reserved 


$\begin{array}{ll}\text { IDE } & \text { Investigational Device Exemption } \\ \text { IGN } & \text { Inferior Gluteal Nerve } \\ \text { Ir } & \text { Iridium } \\ \text { IRB } & \text { Institutional Review Board } \\ \text { LAREI } & \text { Los Amigos Research and Education } \\ & \text { Institute, Downey, CA } \\ \text { LBQ } & \text { Left Buttock Quadrant } \\ \text { LTQ } & \text { Left Thigh Quadrant } \\ \text { MARS } & \text { Metal Artifact Reduction Sequence } \\ \text { NMES } & \text { Neuromuscular Electrical Stimulation } \\ \text { Pavg } & \text { Average Pressure } \\ \text { Pmax } & \text { Maximum Pressure } \\ \text { PPA } & \text { Peak Pressure Area } \\ \text { PU } & \text { Pressure Ulcer } \\ \text { PUP } & \text { Pressure Ulcer Prevention } \\ \text { Qd } & \text { Quadriceps } \\ \text { RBQ } & \text { Right Buttock Quadrant } \\ \text { RF } & \text { Radio Frequency } \\ \text { RLANRC } & \text { Rancho Los Amigos National } \\ & \text { Rehabilitation Center, Downey, CA } \\ \text { ROI } & \text { Region of Interest } \\ \text { RTQ } & \text { Right Thigh Quadrant } \\ \text { Rx } & \text { Treatment } \\ \text { SCI } & \text { Spinal Cord Injury } \\ \text { Sesta-MIBI } & \text { Technetium-99m-MethoxyIsoButyl } \\ & \text { Isonitrile (Cardiolite }{ }^{\circledR} ; \text { Bristol-Myers } \\ \text { SN } & \text { Squibb, N Billerica, MA) } \\ \text { SPECT } & \text { Sciatic Nerve } \\ & \text { Single Photon Emission Computed } \\ \text { Ta } & \text { Tomography } \\ \text { Tl 201 } & \text { Tantalum } \\ & \text { Thallium 201 } \\ & \end{array}$

\section{Introduction}

The review and biomechanical analysis in companion papers I and II [3, 4] highlighted the potential benefits of neuromuscular electrical stimulation (NMES) for pressure ulcer prevention (PUP). These data suggested that long-term, independent activation is required for each of the hip extensor muscle groups: gluteus maximus (GM), innervated by the inferior gluteal nerve (IGN); and hamstrings (HS), innervated by medial branches of the sciatic nerve (SN). In order to be attractive as a prophylactic measure in disabled patients, it is important that this activation be achieved with minimal daily intervention by the patient or caregivers and minimal probability of side-effects or complications. Currently available technologies for NMES cannot meet all of these requirements.

$\mathrm{BIONs}^{\mathrm{TM}}$ (BIOnic Neurons) are injectable, wireless microstimulators that make chronic BION Active Seating (BAS) possible for PUP. BIONs receive power and individually addressed command data by electromagnetic (EM) coupling with an external inductive radio frequency $(\mathrm{RF})$ antenna that must be near the implants (Fig. 1) [9, 15]. Each implant can produce precisely regulated stimulus currents $(0.5-31.5 \mathrm{~mA}$ in $0.5 \mathrm{~mA}$ steps) and pulse durations (8-512 $\mu$ s in $8 \mu$ s steps).

The long-term strategy proposed here is the percutaneous injection of BIONs bilaterally (2 per side) to prevent initial development of pressure ulcers (PUs). This paper presents results from a pilot clinical study in which BION microstimulators were implanted so as to enable selective activation of the GM and HS muscles. Suitable implant sites (see Surgical Procedure below) were identified by cadaver dissections and $\mathrm{CT}$ radiographs to determine nerve sites likely to enable low-threshold, selective stimulation of the target muscles. Three subjects with chronic paraplegia from spinal cord injury (SCI) were implanted unilaterally during gluteal rotation flap reconstruction that they already required for pre-existing Stage III-IV ischial PUs. Although planned as a bilateral treatment ultimately, the contralateral side was not implanted for this initial proof-of-concept trial. Stimulation was applied on a regular basis both to condition the muscles post-operatively (BION Conditioning (BC)), and to unload weight while subjects were seated in their usual wheelchairs (BION Active Seating (BAS)).

\section{Methods}

This pilot clinical trial was conducted at Rancho Los Amigos National Rehabilitation Center (RLANRC, Downey, CA) between Nov 2007 and February 2009, under FDA and IRB approvals (IDE \#G040232, RLANRC/LAREI IRB \#1984, USC IRB \#066001). All subjects gave written informed consent. The trial formed the beginning of a larger study that was designed as a prospective, randomized, controlled trial in SCI subjects $(n=30)$ who were already required to undergo gluteal rotation flap reconstruction for PUs. The IDE permitted non-randomizing/"preloading" the initial 3 subjects into the experimental/treatment arm, to facilitate this pilot exploratory human physiological research presented here. In each subject (desig- 

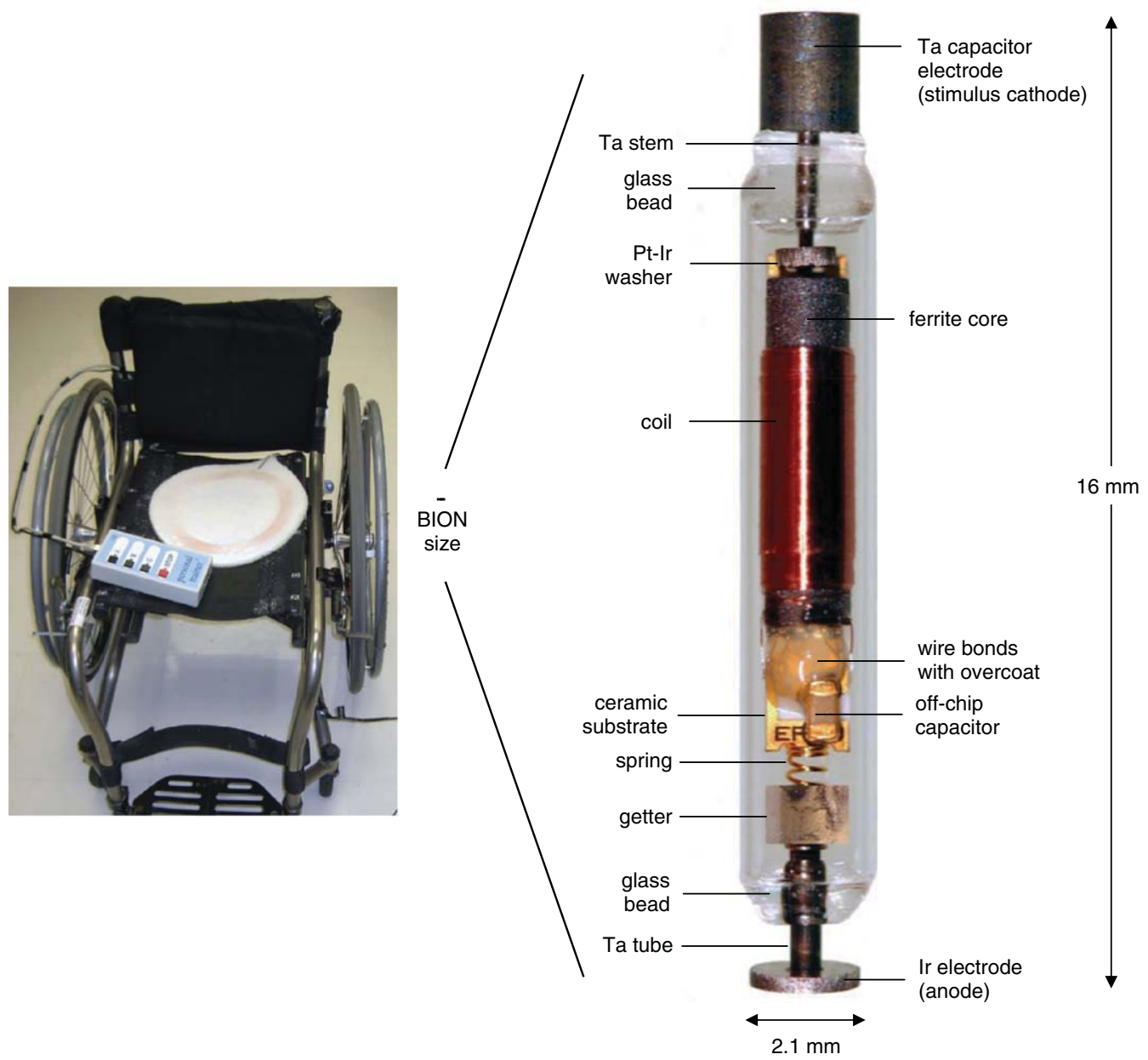

Fig. 1. The BIONTM system (left) may be mounted on a wheelchair or used bedside. It comprises a power source (AC or battery), a "Personal Trainer" (programmable command module), a driver (to generate EM field; not visible), and an external transmission coil (telemeters power and data). The injectable, wireless BION 1-2 neuromuscular microstimulator (right) has a flip-chip ASIC within a black ferrite cylinder. The ferrite core improves inductive coupling by concentrating the EM field from the external coil through the BION's internal coil.

nated PUP1-3) two BIONs were implanted along the IGN and medial SN (to GM and HS, respectively). Outcome measures included: tissue health variables over 6 months (measured by clinical assessment, Xrays, MRI and Sesta-MIBI SPECT muscle perfusion scans); interface pressure redistribution over 6 months (Tekscan, Boston, MA); and recurrence rates over 12 months.

\subsection{Hypotheses (Table 1)}

Primary outcome measures used to assess primary hypothesis: NMES of the HS hip extensors using BIONs would be capable of shifting patients' weights, measured both qualitatively by clinical examination, and quantitatively by shifts in pressure distribution over seated pressure points. Secondary outcome measures used to assess secondary hypotheses: 1) Tissue health would be improved, indexed by: increased muscle perfusion (acutely during stimulation, and chronically at-rest), increased muscle volume, and decreased seated pressure density at-rest, each over a 6 mo. treatment window; as well as decreased PU recurrence rate at 1 year (compared to statistical rates [4]. 2) Wound healing would be improved, indexed by increased flap wound healing and/or decreased complication rates. 3) Surface landmarks would be ascertained, to facilitate optimal placement of BIONs 
Table 1

Trial hypotheses, outcome measures and investigations/parameters (grey = parameters not addressed here due to limited pilot data)

\begin{tabular}{|c|c|c|c|c|}
\hline \multicolumn{2}{|l|}{ Hypotheses } & \multicolumn{2}{|l|}{ Outcome Measure } & \multirow{2}{*}{$\begin{array}{l}\text { Investigation / Parameter } \\
\text { Clinical Examination }\end{array}$} \\
\hline Primary: & & qualitative & visible weight shifting & \\
\hline $\begin{array}{l}\text { HS } \\
\text { NMES }\end{array}$ & Shift Weight & quantitative & shifts in pressure distribution & $\begin{array}{l}\text { Surface Interface Pressure } \\
\text { Mapping }\end{array}$ \\
\hline \multirow{7}{*}{$\begin{array}{l}\text { Secondary: } \\
\text { HS+GM } \\
\text { NMES }\end{array}$} & \multirow{4}{*}{ Improve Tissue Health } & $\begin{array}{l}\text { acute (during stimulation) } \\
\text { chronic (over } 6 \text { mo. follow-up) }\end{array}$ & $\uparrow$ muscle perfusion & $\begin{array}{l}\text { Sesta-MIBI SPECT Perfusion } \\
\text { Scan }\end{array}$ \\
\hline & & \multirow{2}{*}{ chronic (over 6 mo. follow-up) } & $\uparrow$ at-rest muscle volume & MRI \\
\hline & & & $\downarrow$ at-rest pressure density & $\begin{array}{l}\text { Surface Interface Pressure } \\
\text { Mapping }\end{array}$ \\
\hline & & chronic (over 1 yr follow-up) & $\begin{array}{l}\downarrow \text { PU recurrence rate } \\
\text { (compared to statistical rate) }^{1}\end{array}$ & Clinical Examination \\
\hline & \multirow{2}{*}{ Improve Wound Healing } & \multirow{2}{*}{ flap } & $\uparrow$ wound healing rate & Clinical Examination \\
\hline & & & $\downarrow$ wound healing complications & Clinical Examination \\
\hline & Ascertain Surface Landmarks & $\begin{array}{l}\text { facilitate placement of BIONs via } \\
\text { closed injection technique }\end{array}$ & $\begin{array}{l}3 \text { landmark-electrode measures in } \\
\text { each of } 2 \text { planes }\end{array}$ & X-Rays \\
\hline
\end{tabular}

${ }^{1}$ (Kaplan 2010b).

via closed injection in future. The latter two are not addressed here due to limited pilot data.

\subsection{Conduct of study}

Inclusion criteria: 18-70 yo; SCI with complete bilateral lower limb and GM paralysis; at least one Stage III or IV PU scheduled for gluteal rotation flap repair; usually sitting in wheelchair for at least $5 \mathrm{~h} /$ day; otherwise in good health. Exclusion criteria: electronic implants; wound healing concerns; confounding concomitant conditions affecting pelvic region (e.g. another PU not to be corrected by the planned surgery); contraindications for any of the investigations; PUs more than 2.25" deep (to avoid excessive EM coupling distances); usually requiring excessive interventions for spasms that could interfere with NMES. The 3 subjects enrolled were diverse in terms of: gender (1 female and 2 male), age (22-54 yrs), level of injury (C7-T12, complete), time from injury (7-31 yrs), duration of PU (1.5-5 yrs), and BMI (20.8-25.6 kg.m $\left.{ }^{-2}\right)$.

\subsection{Surgical procedure}

During gluteal rotation flap surgery the IGN was identified by palpation of the inferior gluteal artery (with Doppler ultrasound as needed), while the SN was easily exposed (Fig. 2). Their epineuria were not opened. Optimal stimulation sites were identified with a disposable intraoperative nerve stimulator (VariStim $^{\circledR}$ III; Medtronic, Minneapolis, MN). In all cases

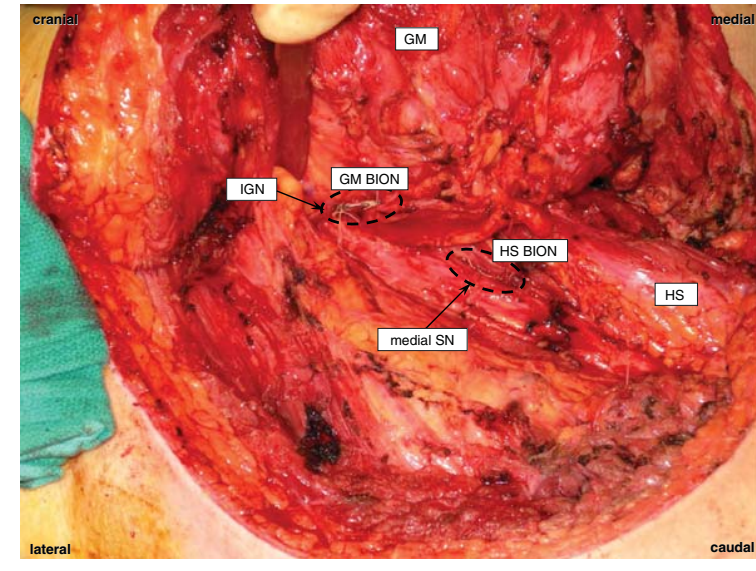

Fig. 2. Intraoperative BION placement.

the HS motor branches lay superficially and medially as the $\mathrm{SN}$ exited the greater sciatic foramen beneath piriformis, as expected [5]. Suitable sites were identified and marked with ink. Each BION was placed with its stimulating Ta capacitor electrode (cathode) distally, closest to its marked location; and its Ir electrode more proximally alongside the nerve. Using $4 / 0$ Vicryl $^{\mathrm{TM}}$ (polyglactin 910; Ethicon, Somerville, NJ) the SN BION was sutured to epineurium and the GM BION to GM fascia, and both were buried in sleeves fashioned from local connective tissue (implemented after PUP2's GM BION, not buried this way, rotated upon sitting, so losing contact with the IGN). All metal was then removed, the flap laid back, and a coil in a sterile transparent drape was brought into the field 
to test both BIONs and record thresholds for muscle twitch. The surgery was then completed as usual, however GM repair was as for a functional muscle (vs. just closing up dead space were a non-functioning one anticipated). Overall surgery was lengthened by $\sim 10-20 \%$ (25-45 min).

\subsection{BION Rx}

All subjects received BION treatment $(\mathrm{Rx})$ which involved 2 phases:

1) BION Conditioning ( $B C)$ : Stimulation of both GM and HS for muscle strengthening, while nonweightbearing; begun once limited movement allowed in 4th week postoperatively (1 week after removal of sutures); aimed for $300 \mathrm{~min} /$ day before BAS started; $60 \mathrm{~min} /$ day thereafter.

2) BION Active Seating (BAS): Stimulation using HS alone to achieve unloading when seated; begun as soon as weightbearing ( $\sim 6$ th week postoperatively); aimed for 300 minutes per day/whenever seated; subjects' trunks and lower limbs safely restrained as needed with strap support behind ankles.

Initial stimulation parameters are defined in Table 2 [6], but were titrated to achieve the pressure relief goals defined elsewhere [6] while avoiding any complications such as excessive loading/motion of distal joints, spasms or autonomic dysreflexia, etc. Subjects received normal nursing pressure care in parallel with BION Rx, including relief of pressure with cushions, regular turnings, etc. The BION Rx schedule was incorporated into their regular in-patient regimen.

\subsection{Investigations and outcome parameters (Table 1)}

\subsubsection{Clinical examination}

Clinical history and examination was performed at all evaluations: BMI, skin color and quality, Braden scale, capillary refill time, PU (stage, healing, recurrence), deep tendon reflexes (to ensure intact $\mathrm{SN}$ preoperatively), unloading during BAS, compliance (based on diary and usage data recorded by BION system), subject satisfaction.

\subsubsection{Surface interface pressure mapping \\ 2.5.2.1 Rest mapping}

At all evaluations at which subjects were able to sit, pressure distributions were recorded at rest using the CONFORMat ${ }^{\circledR}$ System (Tekscan, Boston, MA) (Fig. 3). To improve accuracy and repeatability, a standardized setup was used [13]: subjects seated in adjustable wheelchair; thighs flat; hips, knees, ankles, elbows flexed to $90^{\circ}$; strap behind ankles. Sensor mat $1.8 \mathrm{~mm}$ thick, $47 \mathrm{~cm} \times 47 \mathrm{~cm}, 32 \times 32$ array of sensors; between subject and standardized $10.2 \mathrm{~cm}$ thick high-density foam cushion $\left(45 \mathrm{~kg} . \mathrm{m}^{-3}\right)$ on flat hard board. Each of 1,024 ink-resistive sensing elements provide spatial resolution of $1.5 \times 1.5=2.25 \mathrm{~cm}^{2}$ per cell; sensitivity range $0-34.0 \mathrm{kPa}(0-255 \mathrm{mmHg})$ at an amplitude resolution of $0.13 \mathrm{kPa}(1 \mathrm{mmHg})$; calibrated accuracy of $\pm 3 \%$ [14].

Seat pressures at rest were analyzed by defining 3 normalized variables: $\mathrm{Pavg}=$ the average pressure

Table 2

Initial stimulation parameters

\begin{tabular}{|c|c|c|c|c|}
\hline \multicolumn{2}{|c|}{ Stimulation Parameter } & \multirow{2}{*}{$\begin{array}{l}\text { Effect } \\
\text { Recruitment }\end{array}$} & \multirow{2}{*}{$\begin{array}{l}\text { Bion Conditioning }(\mathrm{BC}) \\
\mathbf{3}\end{array}$} & \multirow{2}{*}{$\begin{array}{l}\text { BION Active Seating (BAS) } \\
\mathbf{2 - 4}\end{array}$} \\
\hline Stim Strength & TU & & & \\
\hline Pulse Width $\times$ Pulse & Threshold & & High to recruit all fibers. & High to recruit all fibers. \\
\hline Amplitude $(\mathrm{ms} \times \mathrm{mA})$ & Units (nC) & & & \\
\hline \multirow[t]{4}{*}{ Frequency } & \multirow[t]{4}{*}{$\mathbf{H z}$} & \multirow[t]{4}{*}{ Force } & $1-5$ & $10-30$ \\
\hline & & & Seeking unfused, isolated twitches. & Seeking wide dynamic range to \\
\hline & & & Low $\mathrm{f}$ of action potentials (APs) & maximize motion. \\
\hline & & & to initiate lower force contractions. & $\begin{array}{l}\text { High } \mathrm{f} \text { of APs to achieve full } \\
\text { contractions/tetany. }\end{array}$ \\
\hline \multirow[t]{4}{*}{ Duty Cycle } & \multirow[t]{4}{*}{$\mathbf{s}$} & \multirow[t]{4}{*}{ Exercise } & $50 \%$ & $25 \%$ \\
\hline & & & ( $5 \mathrm{~s}$ on, $5 \mathrm{~s}$ off $)$ & ( $5 \mathrm{~s}$ on, $15 \mathrm{~s}$ off $)$ \\
\hline & & & Mimics normal repetitive exercise. & Adequate weight shifting while \\
\hline & & & $\begin{array}{l}\text { Lower force contractions to avoid } \\
\text { fatigue or compromising blood supply. }\end{array}$ & $\begin{array}{l}\text { avoiding disturbing movements. } \\
\text { Adequate rest to avoid fatigue } \\
\text { or compromising blood supply. }\end{array}$ \\
\hline
\end{tabular}


(a) PUP1

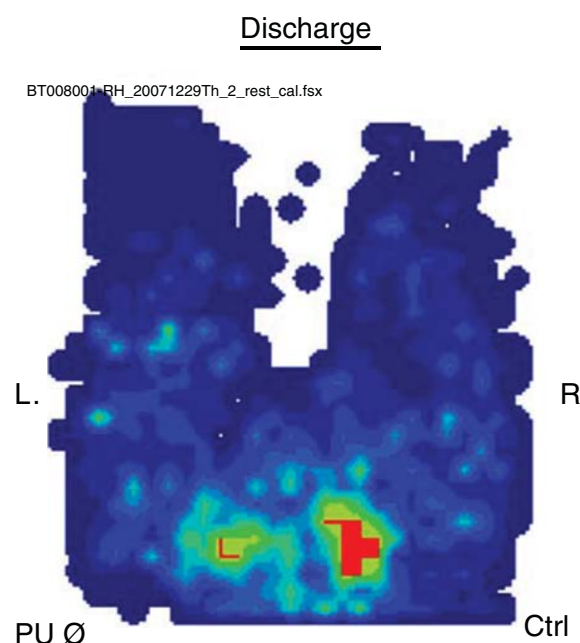

(b) PUP3

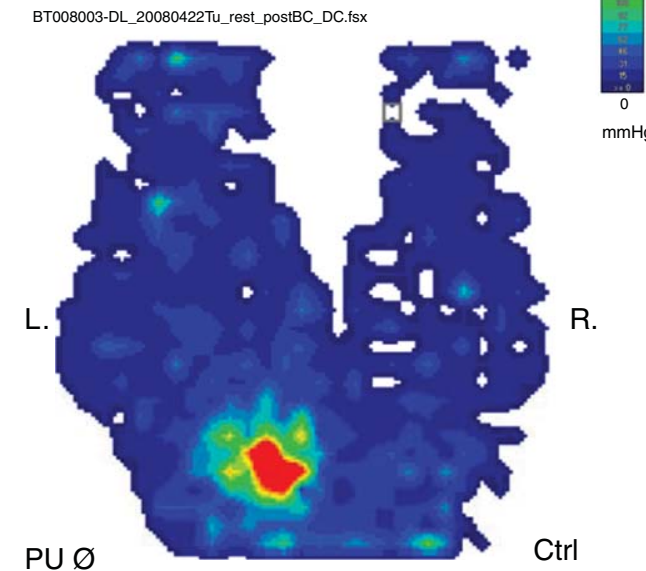

\section{6 mo.s BION Rx (avg 107 min/day)}

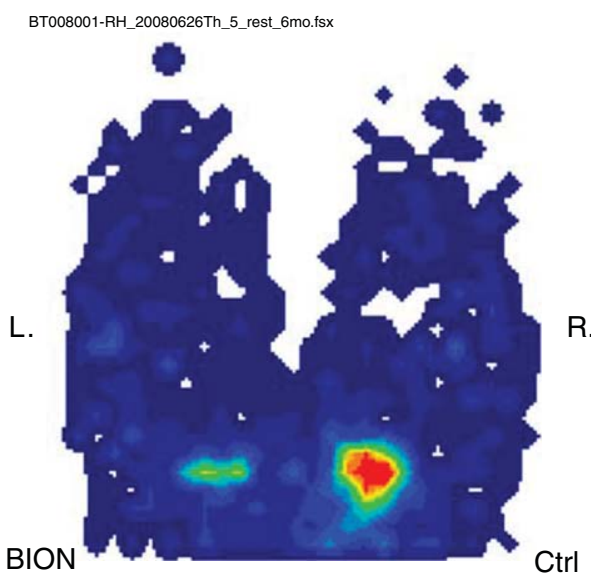

6 mo.s BION Rx (avg $187 \mathrm{~min} /$ day)

BT008003-DL_20081028Tu_5_rest_6mo.fsx

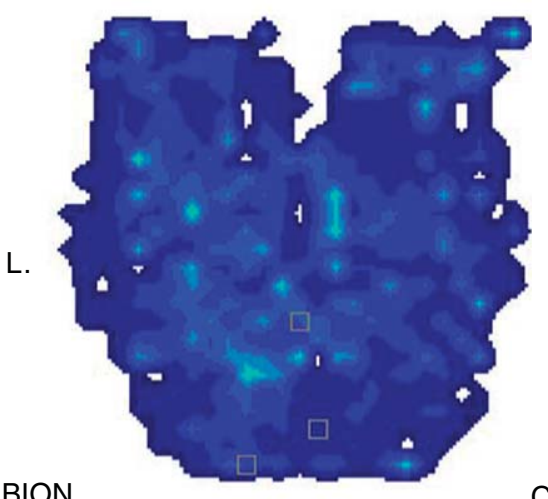

Ctrl

Fig. 3. Surface interface pressure maps at rest for the 2 subjects that completed the study protocol, upon discharge (left) and after 6 mo. BION Rx window (right). (a) PUP1 averaged $107 \mathrm{~min} /$ day BION Rx; (b) PUP3 averaged $187 \mathrm{~min} /$ day. PU repair and implant sides are on the L. in each case. Comparisons with the unoperated control (Ctrl) sides are useful: High pressures on the PU side at discharge become much reduced and more diffusely distributed with BION Rx.

in the contact area of each quadrant; Pmax $=$ the maximum pressure in each quadrant; PPA (Peak Pressure Area) $=$ the area in each quadrant with pressures exceeding $60 \mathrm{mmHg}$ (as an indicator of likely capillary occlusion [3]. To facilitate intersubject comparisons, each quadrant's Pavg and Pmax were normalized to the average pressure across the full contact area; and each quadrant's PPA was normalized to the overall contact area across all 4 quadrants. For each, the $\%$ change from the value at discharge (DC) was plotted over the course of the 6 mo. BION Rx window (Fig. 4).

\subsubsection{Dynamic mapping}

For dynamic mapping during NMES the CONFORMat ${ }^{\circledR}$ System could not be used because the RF EM field required to energize the BION implants interfered with the ink-resistive sensors. An alternative system based on capacitive sensors (XSensor ${ }^{\mathrm{TM}}$ X36 System; XSensor Technology, Calgary, AB, Canada) [3] was explored but could not be used because its conductive plane blocked the RF energy. A rudimentary pneumatic system was therefore fashioned to offer some qualitative sense of dynamic pressure changes 

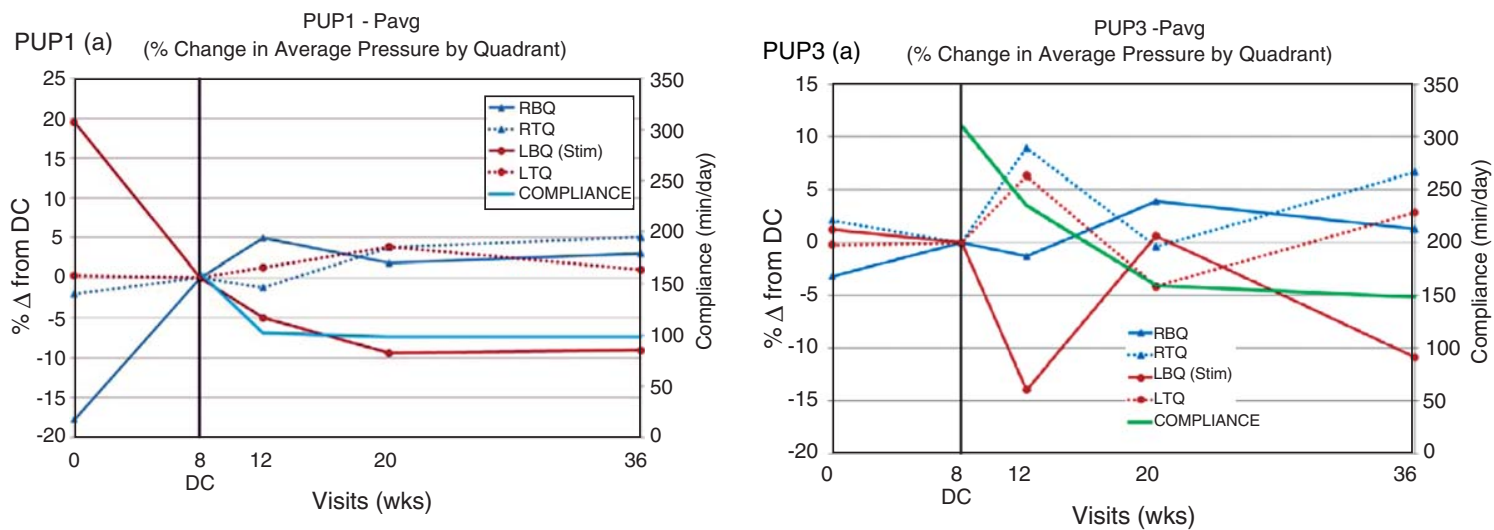

PUP1 -Pmax
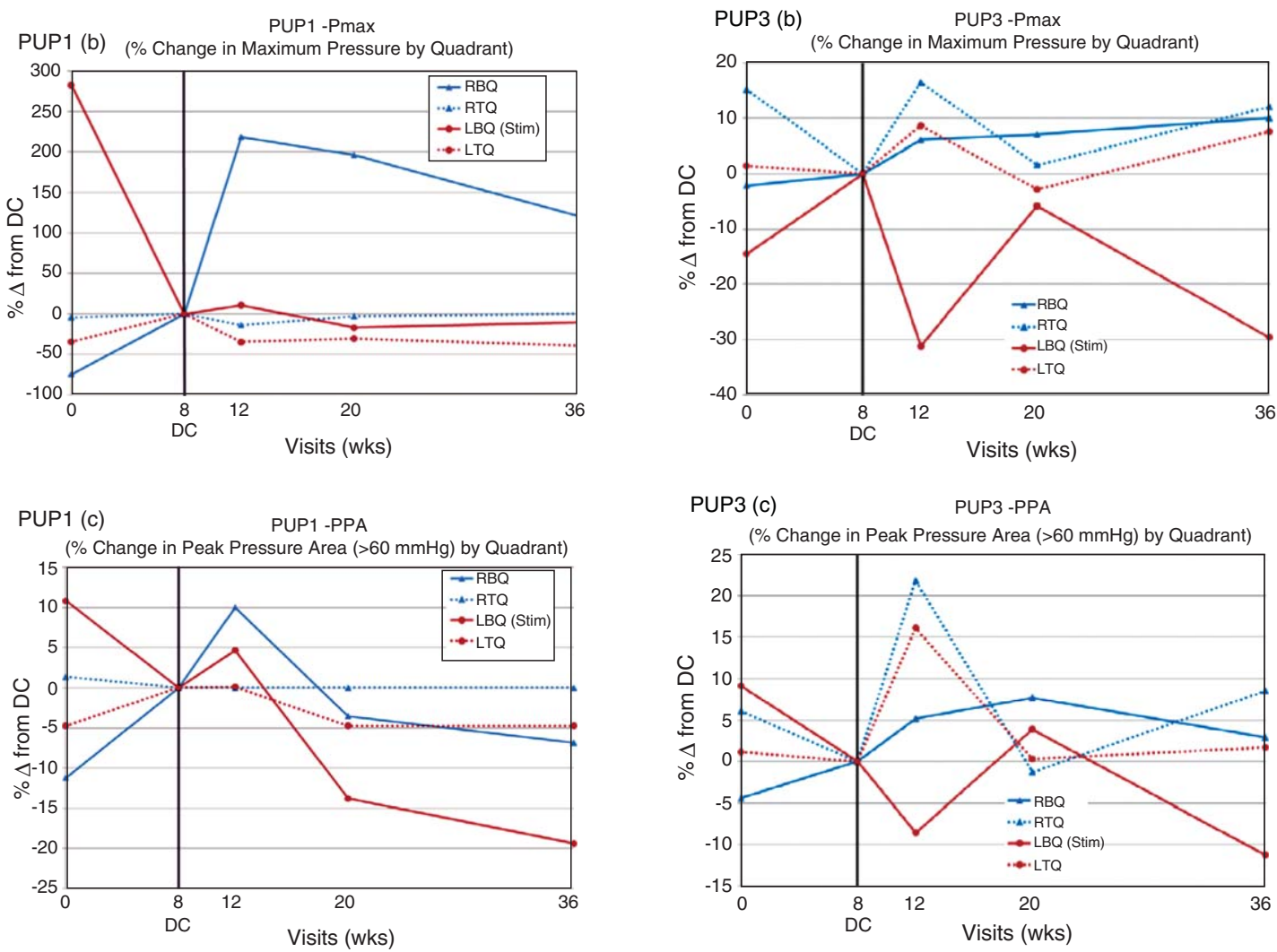

Fig. 4. Surface interface pressures for left and right buttock and thigh quadrants (LBQ, RBQ, LTQ, RTQ), from before surgery through the 6 mo. Rx window after discharge (DC), in subjects PUP1 and PUP3. LBQ was the implanted and stimulated side in both cases. Each curve represents the \% change relative to the value at the time of DC. Compliance is shown in panels (a) only, but is relevant to (b) and (c) too (from Fig. 9). (a) Pavg: Average pressure in contact area of each quadrant, normalized to average pressure across full contact area. LBQ (activated side) improved by Pavg $-9.1 \%$ in PUP1 and $-10.9 \%$ in PUP3 (vs. RBQ $+3.0 \%$ and $+1.3 \%$ ). (b) Pmax: Maximum pressure in each quadrant, normalized to average pressure across full contact area. LBQ (activated side) improved by Pmax $-10.3 \%$ and $-29.6 \%$ (vs. RBQ $+120.2 \%$ and $+10.0 \%$ ). (c) PPA: Peak Pressure Area is area in each quadrant with pressures exceeding $60 \mathrm{mmHg}$ (see text), normalized to full contact area. LBQ (activated side) improved by PPA $-19.4 \%$ and $-11.2 \%$ (vs. RBQ $-6.9 \%$ and $+2.9 \%$ ).

achieved during BAS (Fig. 5): Pneumatic bladders (pediatric BP cuffs) beneath each quadrant fed into pressure transducers (SX05 N; SenSym ICT, Milpitas,
CA), which analogue output was amplified (INA121; Burr-Brown Corp, Tucson, AZ), and sampled digitally at $1 \mathrm{kHz}$ in LabVIEW ${ }^{\mathrm{TM}}$ (National Instruments Corp, 
(a)

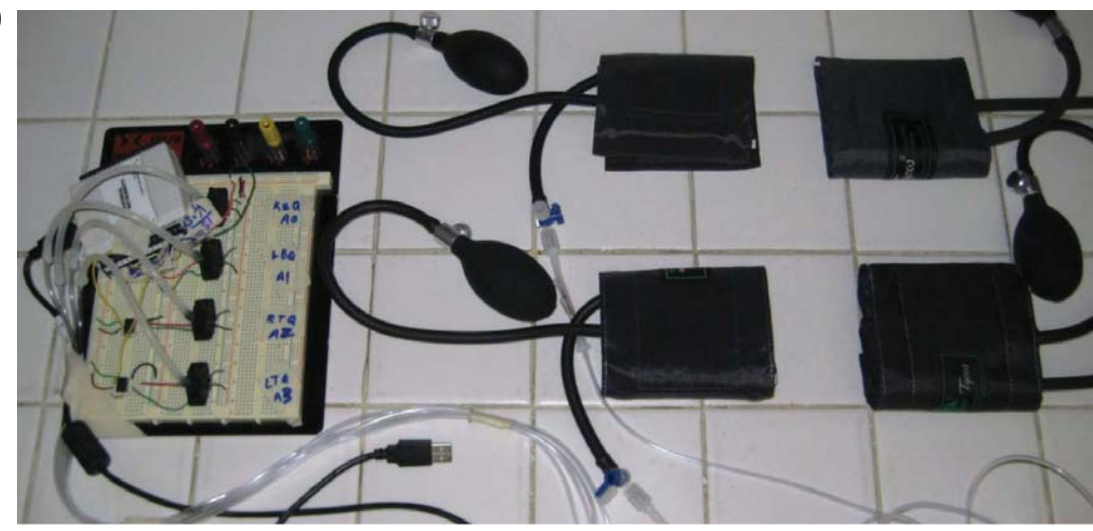

(b)

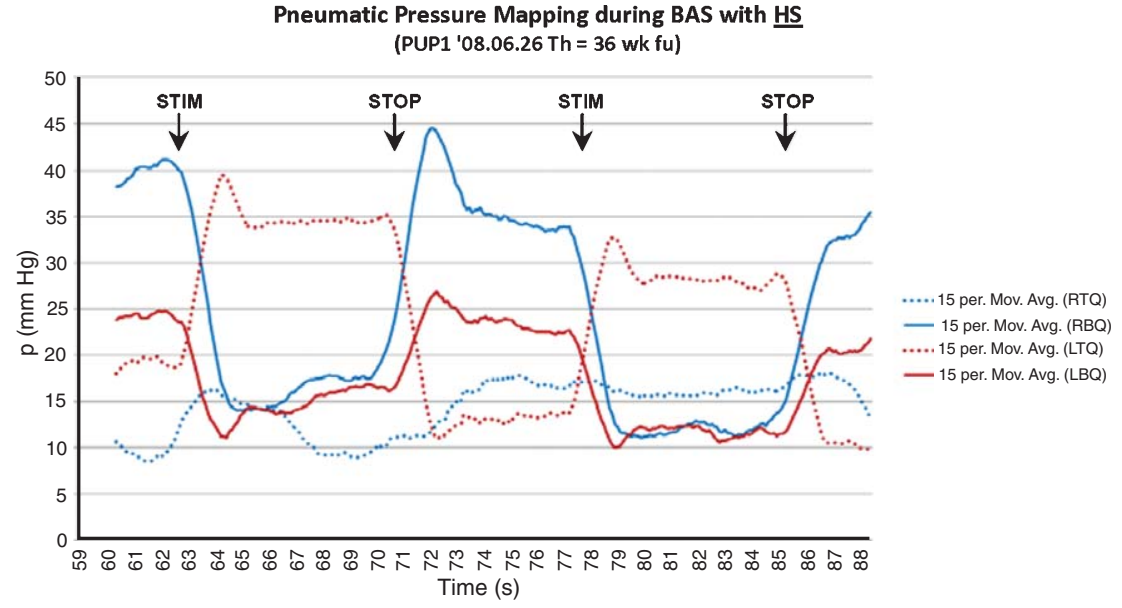

(c)

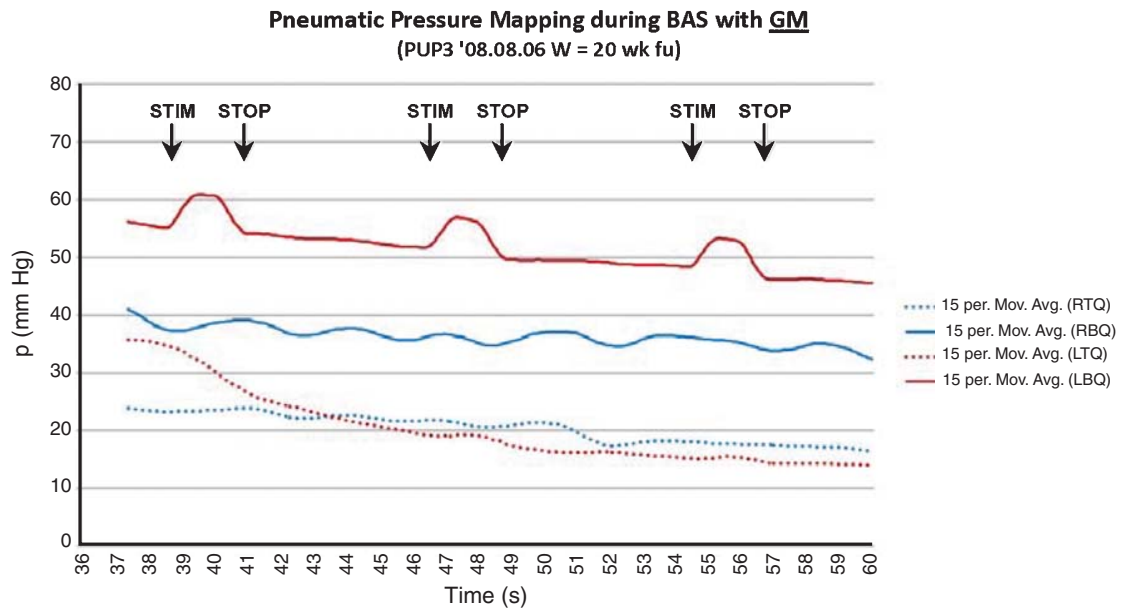

Fig. 5. Rudimentary pneumatic system fashioned to obtain some sense of dynamic pressure changes during BAS (a). Pneumatic bladders (pediatric BP cuffs) beneath each quadrant feed into pressure transducers, amplified, and sampled at $1 \mathrm{kHz}$ (LabVIEWTM). Examples of BAS using HS stimulation in PUP1 (b) and GM stimulation in PUP3 (c). During HS stimulation of LBQ (b), pressures fall beneath both buttocks to as low as $10 \mathrm{mmHg}$, taken up predominantly by ipsilateral thigh quadrant (LTQ). Pressure beneath RBQ is higher than pressure beneath LBQ both before and during (to a lesser degree) stimulation. During GM stimulation of LBQ (c), pressures rise ipsilaterally, while variations beneath contralateral RBQ may indicate shifting. 
Austin, TX). The same standardized setup was used as described for Rest Mapping above.

\subsubsection{Sesta-MIBI SPECT perfusion scans}

Sesta-MIBI SPECT perfusion scintigraphy was performed to assess changes in GM and HS perfusion over 6 mo. BION Rx window (Fig. 6). Intravenous Tc-99 Sesta-MIBI radiotracer (Technetium-99mMethoxyIsoButyl Isonitrile (Cardiolite ${ }^{\circledR}$ ); BristolMyers Squibb, N. Billerica, MA) was injected; redistribution into mitochondria (proportional to muscles' perfusion) was measured by Single Photon Emission Computed Tomography (SPECT), using Philips Axis 2 VT scintigraphy scanner with Odyssey
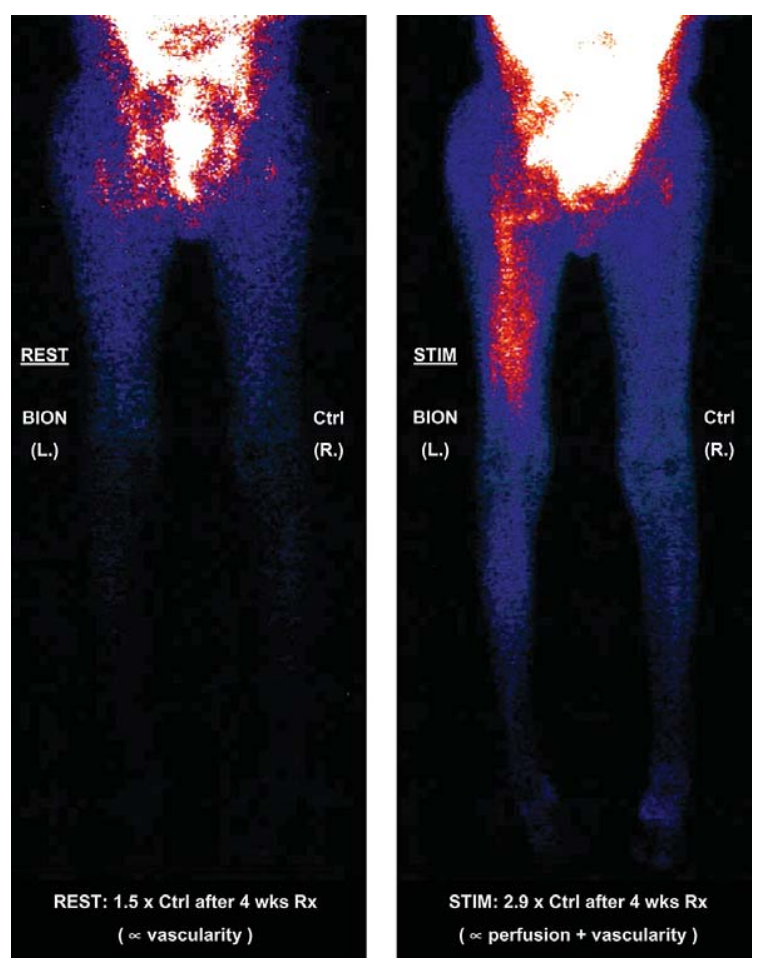

Fig. 6. Examples of Sesta-MIBI SPECT muscle perfusion scans, from behind, after 4 weeks BION Rx (PUP1): at rest (left), and during BION NMES of HS only (right). Images should not be compared to each other as different amounts of radio-isotope are injected for the different scans, and scans are at different decay times. However, comparing L. and R. thighs in each panel is useful to evaluate BION $\mathrm{Rx}$ vs. control (Ctrl) sides in each state. Counts are normalized (see text), and effects of surgery have passed (as evidenced by almost equal GM counts at rest bilaterally in subjects who have not received GM Rx; and HS unaffected by surgery in any event). HS effects are therefore due to BION Rx alone. In this case perfusion after 4 weeks of $\mathrm{BC}$ is $1.5 \times \mathrm{Ctrl}$ at rest ( $\propto$ vascularity, i.e. chronic effect); $2.9 \times$ Ctrl during stimulation ( $\propto$ perfusion in addition to vascularity).
LX software (Philips Medical Systems, Bothell, WA); resolution $\pm 5 \%$ perfusion change (personal communication: ME Siegel, Dept of Nuclear Medicine, USC, Los Angeles, CA, Dec 2003) [11]. Tomograms were quantified by determining average count per area in regions of interest (ROIs) of axial slices through relevant muscles (Fig. 7). These counts were normalized against average counts from contralateral quadriceps (Qd; selected as this was an unactivated muscle group on the non-stimulated side). Rest data was determined from a rest scan:

$$
\text { rest_val } m=\frac{\text { rest_cnt }}{\text { rest_cnt }_{c t r l}}
$$

where rest_val $=$ final rest ratio, rest_cnt $=$ average count at rest, $m=$ muscle (GM, HS or Qd), and ctrl = control (contralateral Qd).

Stimulation data was determined from the difference between a stimulation scan (injection during final 2 min of 6 min of maximal BION Rx), and a preceding scout scan (resting baseline of remaining radiation from earlier rest scan):

$$
s t i m_{-} v a l_{m}=\frac{(\text { stim_cnt }- \text { scout_cnt })_{m}}{(\text { stim_cnt }- \text { scout_cnt })_{c t r l}}
$$

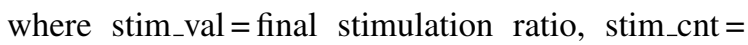
average count during stimulation, scout_cnt = average count during scout scan, $m=$ muscle (GM, HS or Qd), and $\mathrm{ctrl}=$ control (contralateral Qd).

As described under Results, the rest_val parameter was used as an indicator of vascularity, while the stim_val parameter was used as an indicator of perfusion.

\subsection{4. $M R I$}

Standard MRIs were used to assess changes in muscle volume over 6 mo. BION Rx window. T2 weighted axial and sagittal scans from iliac crests to knees, using GE Sigma 1.5 T scanner (GE Medical Systems, Waukesha, WI); $8 \mathrm{~mm}$ slices, $2 \mathrm{~mm}$ spacing; MARS (Metal Artifact Reduction Sequence) protocol to minimize BION artifacts. Indices of muscle volume were determined using each muscle's maximal thickness (T), maximal width (W), and origin-insertion length (L). Resultant volume index normalized to contralateral Qd (unactivated muscle on non-stimulated side):

$$
\text { vol_index }_{m}=\frac{(T \cdot W \cdot L)_{m}}{(T \cdot W \cdot L)_{c t r l}}
$$




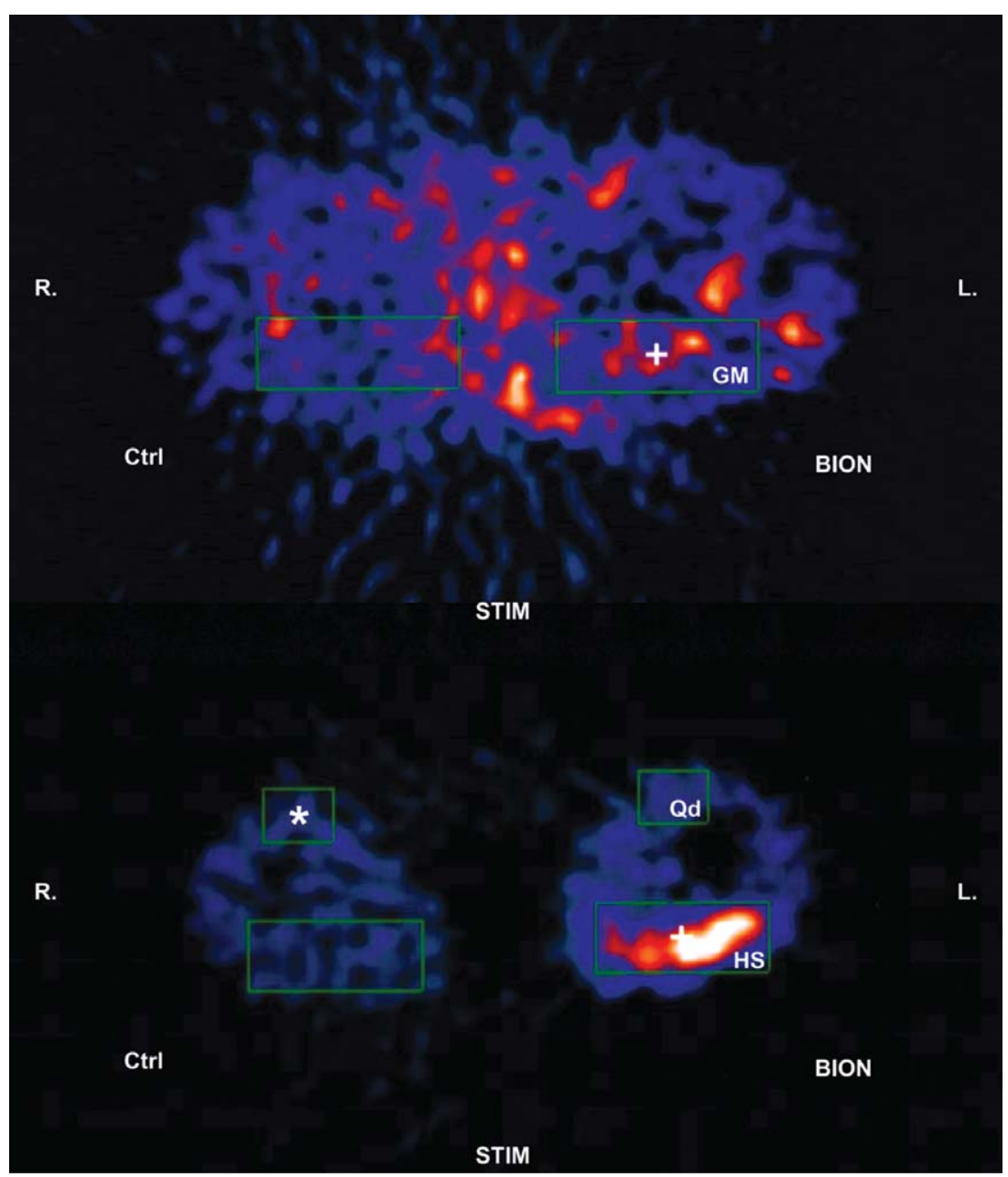

Fig. 7. Scintigraphy counts in axial tomography slices, shown from inferiorly, with regions of interest (ROIs) defined through GM (above) and HS+Qd (below), during BION Rx of HS only. Counts normalized to uninvolved Qd on non-stimulated side $\left(^{*}\right)$.

where vol_index=normalized index of volume, $T=$ maximal muscle thickness, $W=$ maximal muscle width, $L=$ origin-insertion length, $m=$ muscle (GM, HS or Qd), and ctrl=control (contralateral Qd).

\subsubsection{X-rays}

Pelvic X-rays (seated lateral, and prone orthogonal (AP + cross-table lateral)) taken to assess "padding thickness" between ischial tuberosity and seat (during loading) or surface (when prone); and to determine BIONs' positions relative to palpable bony landmarks (for eventual closed implantation; not discussed further here due to limited data).

\section{Results}

\subsection{Experience with implants}

In all subjects two BIONs were implanted successfully at the desired locations, one on each nerve; and selective activation of GM and HS was achieved intraoperatively and postoperatively (threshold stimulus levels in Fig. 8). PUP1's GM BION failed shortly after postoperative activation. PUP2's GM BION rotated upon first sitting, such that GM activation could no longer be achieved. Subsequent PUP3 GM BION was secured to fascia rather than epineurium, and both BIONs were buried alongside their nerves in sleeves 


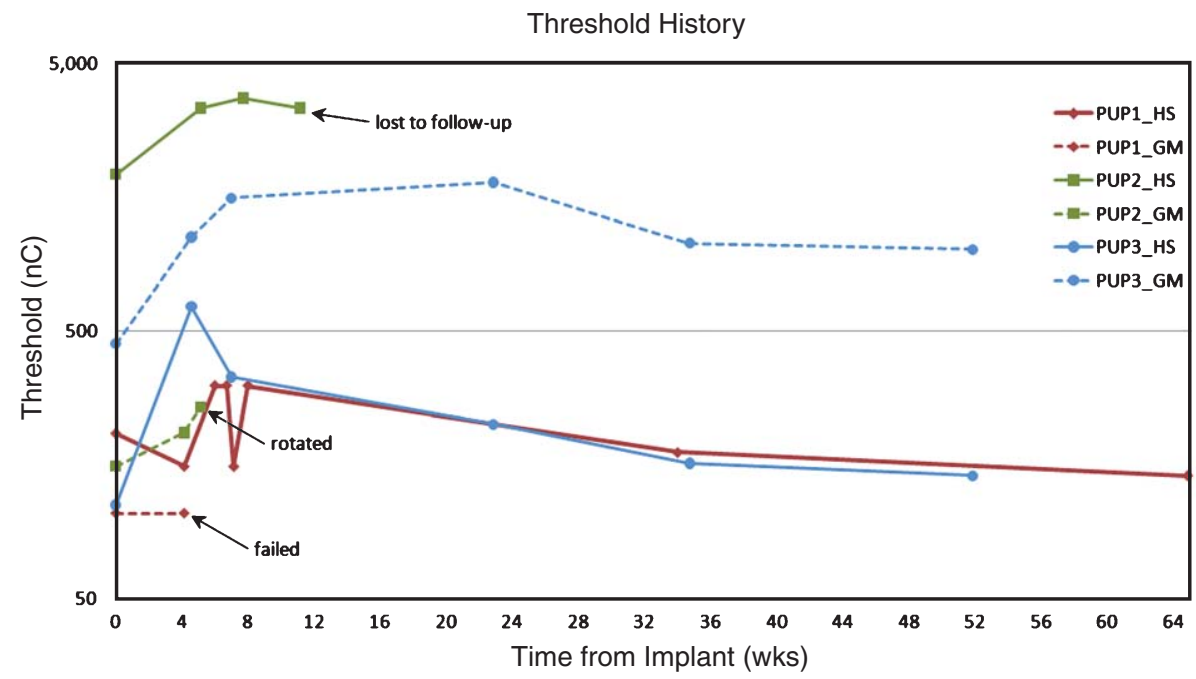

Fig. 8. Log threshold plots for the 6 implanted BIONs: Threshold $(\mathrm{nC})=$ Pulse Amplitude $(\mathrm{mA}) \times$ Pulse Width $(\mu \mathrm{s})$. Acceptably low thresholds throughout, with classic initial increase due to wound healing and capsular formation, settling progressively over 3-6 months. PUP1: GM BION failed early (4 wks). PUP2: GM BION moved upon initial sitting (6 wks); PUP2 later lost to follow up.

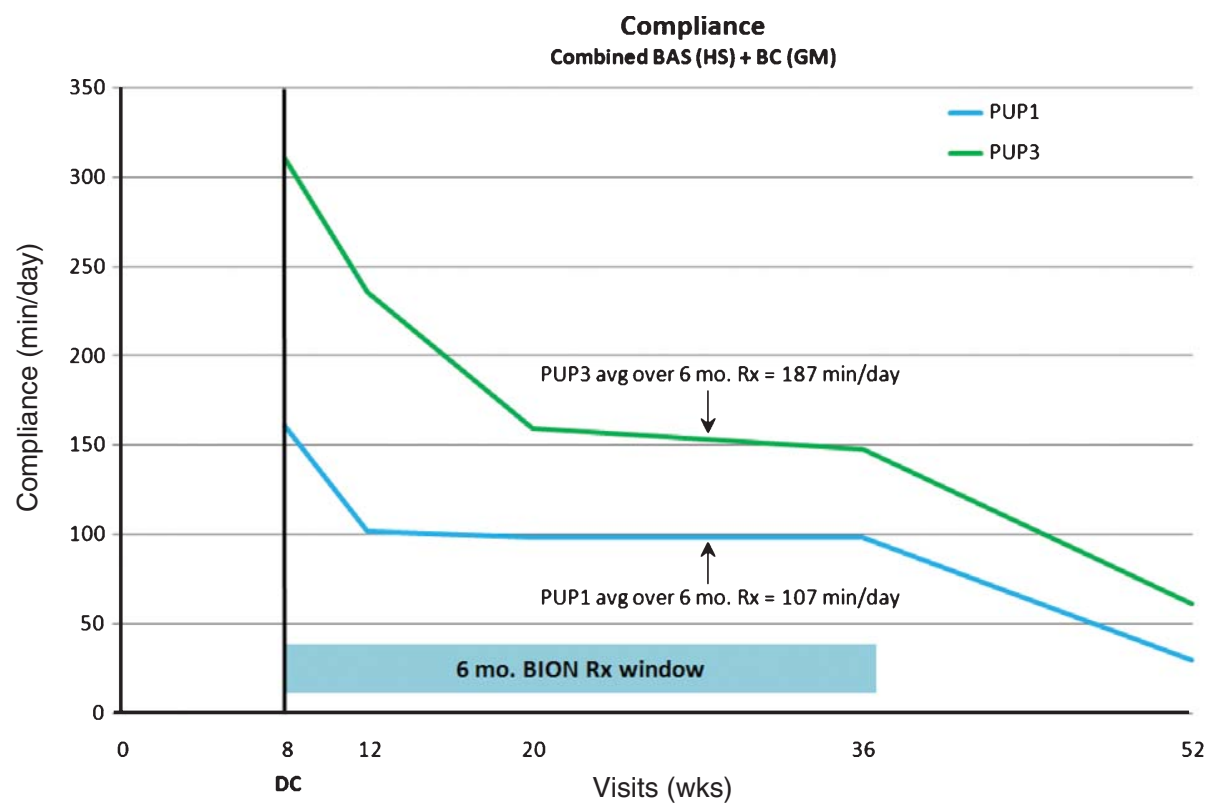

Fig. 9. Compliance shown as combined BION Rx $(\mathrm{BAS}+\mathrm{BC})$, in minutes per day, for each subject who completed the study protocol. For both, compliance was adequate in first 6 months (associated with more frequent follow-ups and required investigational visits). This period was used as a consistent treatment window over which parameters were compared throughout (average BION Rx = $147 \mathrm{~min} / \mathrm{day}$ (107 in PUP1; 187 in PUP3)).

of local connective tissue. In PUP2 and PUP3, scar tissue surrounding the nerves limited the proximity with which BIONs could be placed to their targets, resulting in somewhat higher thresholds.

\subsection{Experience with treatment}

PUP1 and PUP3 completed the full 12 mo. protocol; PUP2 withdrew after 5 months due to personal 
life changes. Mean daily use of the stimulation system over time is plotted in Fig. 9. Compliance was limited by the system's requirement for $\mathrm{AC}$ power. A battery-powered prototype system was trialed temporarily to address the mobility limitation, and achieved 300+ min/day compliance but was withdrawn due to technical problems.

\subsubsection{Side-effects of stimulation}

At enrollment PUP2 was chronically medicated for spasms, and noted same in the contralateral limb, at their usual severity, coincident with the onset of stimulation. Increased ramp-up times led to their passing after an initial 2 or 3 contractions in each session. In PUP1 vigorous BAS ( $>3 \mathrm{x}$ threshold) resulted in plantar flexion and ankle inversion. In PUP3 crossstimulation of gastrocnemius occurred with all GM stimulation (indicating proximity to $\mathrm{SN}$ ), and with $>2 \mathrm{x}$ threshold HS stimulation. This had not been noticed intra-operatively, where the legs were covered in drapes and only threshold stimulations were sought. In future this should be actively excluded by stimulating well above threshold while concurrently palpating and visually assessing distal muscle groups.

\subsubsection{Adverse events}

PUP1 fell asleep on the coil driver, which radiates substantial heat during continuous use. A small blister developed on their thigh, which was discovered coincidently and healed rapidly and without incident. Subsequently longer cables were implemented so that the driver could lie on the floor.

\subsection{Outcome measures}

\subsubsection{Clinical examination}

In all subjects BMI remained constant throughout, and improvements were noted in skin color and quality, Braden Scale, and capillary refill time. All surgical wounds healed without complications. All subjects demonstrated movement and shifting of weight during BAS. No PUs occurred or recurred. All subjects enjoyed BION Rx, other than being tethered to $\mathrm{AC}$ power.

\subsubsection{Surface interface pressure mapping \\ 3.3.2.1 Rest mapping}

Figure 3 shows rest maps upon DC and after the 6 mo. window of BION Rx, for both subjects who completed the study. High pressures on the PU side at DC became much reduced and more diffusely distributed with BION Rx, particularly in the more compliant PUP3.

Figure 4 plots the rest pressure data for these same 2 cases, for each quadrant, from before surgery, through the 6 mo. Rx window after DC. Each curve represents the $\%$ change relative to the value for that parameter at the time of DC. Over the Rx period, the Left Buttock Quadrant (LBQ; Rx side) improved by Pavg -10\% (9\% and $-11 \%$ for PUP1 and PUP3 respectively), Pmax $-20 \%(-10 \%$ and $-30 \%)$, and PPA $-15 \%(-19 \%$ and $-11 \%)$; while pressures on the untreated contralateral Right Buttock Quadrant (RBQ) deteriorated by Pavg $+2 \%(+3 \%$ and $+1 \%)$ and Pmax $+65 \%(+120 \%$ and $+10 \%)$, with a slight improvement in PPA $-2 \%(-7 \%$ and $+3 \%)$.

\subsubsection{Dynamic mapping}

Figure 5 shows examples of BAS using HS stimulation vs. GM stimulation. During HS stimulation on the L. side, pressures fell beneath both buttocks, being taken up by the ipsilateral Left Thigh Quadrant (LTQ) predominantly. Using GM stimulation, on the other hand, pressures rose under the LBQ during stimulation, while variations beneath the contralateral RBQ may have been indicative of shifting on that side.

\subsubsection{Sesta-MIBI SPECT perfusion scans}

Figure 6 shows example perfusion scans after only 4 weeks BION Rx (PUP1). Rest scan data is particularly important as it indicates a chronic effect on vascularity. The combined data for both subjects over the full 6 mo. $\mathrm{Rx}$ window indicated that vascularity was increased by $1.2 \times$ in GM (PUP3 only), and an average of $2.1 \times$ in HS (3.0 $\times$ PUP1; $1.3 \times$ PUP3). Stimulation data indicates overall maximal perfusion (including effects of both vascularity and vasodilation). The combined data indicated that perfusion was increased by $1.7 \times$ in GM (PUP3 only), and an average of $5.4 \times$ in HS $(7.0 \times$ PUP1; 3.8× PUP3).

\subsection{4. $M R I$}

Over the 6 mo. period MRI muscle volume was increased by an average of $+30.5 \%$ in HS $(+24.4 \%$ PUP1; $+36.6 \%$ PUP3), and by $+13.8 \%$ in GM (PUP3 only), based on the muscle volume index metric described under Methods. 
Table 3

Increase on X-ray of soft-tissue padding thickness over 6 mo. BION Rx

\begin{tabular}{|c|c|c|c|c|c|c|}
\hline \multirow[t]{2}{*}{ X-ray } & \multicolumn{2}{|r|}{ PUP1 } & \multicolumn{2}{|r|}{ PUP3 } & \multicolumn{2}{|c|}{ COMBINED AVG $(n=2)$} \\
\hline & $\begin{array}{c}\% \\
\text { incr. }\end{array}$ & $\begin{array}{l}\text { initial thickness + } \\
\text { increase over } 6 \text { mo. }\end{array}$ & $\begin{array}{c}\% \\
\text { incr. }\end{array}$ & $\begin{array}{l}\text { initial thickness }+ \\
\text { increase over } 6 \text { mo. }\end{array}$ & $\begin{array}{c}\% \\
\text { incr. }\end{array}$ & $\begin{array}{l}\text { initial thickness + } \\
\text { increase over } 6 \text { mo. }\end{array}$ \\
\hline Loaded (seated) & $47 \%$ & $1.5+0.7 \mathrm{~cm}$ & $64 \%$ & $1.4+0.9 \mathrm{~cm}$ & $55 \%$ & $1.5+0.8 \mathrm{~cm}$ \\
\hline Unloaded (prone) & $18 \%$ & $3.3+0.6 \mathrm{~cm}$ & $67 \%$ & $2.7+1.8 \mathrm{~cm}$ & $42 \%$ & $3.0+1.2 \mathrm{~cm}$ \\
\hline Average & $32 \%$ & $2.4+0.7 \mathrm{~cm}$ & $65 \%$ & $2.1+1.4 \mathrm{~cm}$ & $49 \%$ & $2.2+1.0 \mathrm{~cm}$ \\
\hline
\end{tabular}

\subsubsection{X-rays}

On X-ray, soft-tissue padding thickness increased over the 6 mo. period by an average $+49 \%(1 \mathrm{~cm})$ (Table 3).

\section{Discussion}

BAS is intended ultimately as a bilateral and preventive treatment, potentially to benefit both ischial and sacral PUs. These constitute $45 \%$ of PUs in SCI, of which only $11 \%$ are amenable to surgery (Stage IIIIV) [8]. The pilot trial detailed here took advantage of the open access afforded by surgery that these patients required anyway. Although this approach only offered unilateral access, the resultant study model had the following advantages: 1 ) access was readily available, allowing for precise BION placement and so reducing confounding variables related to optimal electrode location (minimizing charge required for stimulation and risk of cross-stimulation of nearby nerves); 2) each patient acted as their own intra-patient control; 3) patients requiring PU repair are at especially high-risk of recurrence (constituting a particularly good group to assess the efficacy of a preventive treatment); 4) implantation was only a relatively minor addition to the major surgery scheduled (vs. the alternative of percutaneous placement into undamaged tissues before any proof-of-concept).

\subsection{Pressure mapping}

Despite such limited data, clear improvements in rest pressures on the treatment side appear to be associated with some deterioration on the untreated side: The contralateral Pavg and PPA remained largely unchanged over the 6 mo. window ( $\pm 2 \%$ ), while Pmax increased considerably $(+65 \%)$. It is unlikely that this increase in Pmax represents shifting of weight to the contralateral side due to BION Rx ipsilaterally, as if that were the case, the contralateral Pavg and PPA would also have been expected to deteriorate, but did not. Rather, this may reflect the common clinical pattern of regression towards PU recurrence on the untreated side, as a high pressure point continued to protrude through still-atrophic tissues.

\subsection{Muscle perfusion}

Researchers in the field of NMES for PUP have utilized a variety of outcome parameters for assessing improvements in tissue health. These include, for example, transcutaneous $\mathrm{O}_{2}$ measurements [2], thermography [10], and more recently dynamic CT or MRI studies [12]. In considering which would best serve our purposes it was noted that, although the transcutaneous modalities are easy to perform and non-invasive, they provide estimates of cutaneous blood flow only rather than measuring deep muscle perfusion [7]. Sesta-MIBI SPECT perfusion scans are widely used for assessing cardiac muscle perfusion, and scanning for tumors; and less so for assessing peripheral vascular disease [11]. However, to date this technique has not been widely used for assessing limb muscle perfusion. The initial results presented here demonstrate that this may be a useful new application of a long-standing modality.

Another popular perfusion scanning technique that was considered is Thallium 201 ( $\mathrm{Tl} 201$ ) scintigraphy. Tl 201 enters cells by $\mathrm{Na}^{+} / \mathrm{K}^{+}$ATPase pump activity, however, and so is dependent not only on perfusion, but also on functioning muscle. Sesta-MIBI, on the other hand, is taken up by mitochondria in proportion to perfusion, more than function per sé. Furthermore, Tl 201 uptake is measured by planar scintigraphy, rather than SPECT, and so could have resulted in muscle activity being obscured by overlapping tissues [11].

The distinction should be made between improvements in perfusion associated with: 1) chronic increases in vascular capacity due to muscle hypertrophy = "vascularity"; and 2) acute increases in 
vascular flow due to vasodilation during muscle activation = "perfusion". While stimulation scan results represent both (the acute changes in perfusion on top of the chronic changes in vascularity), rest scan results represent only the chronic effects on vascularity/hypertrophy. As the confounding effect of vasodilation is excluded at rest, it was believed that the rest results, rather than the stimulation results, were an especially significant indicator of long-term improvement.

\subsection{Adverse events}

Patients on treatment for excessive spasms or autonomic dysreflexia were excluded from enrollment in this study. Furthermore, for well-placed electrodes on or near a mixed peripheral nerve such as the SN, relatively low currents and pulse widths, at low frequencies, are required to activate the large-diameter, myelinated $\alpha$ motor neurons to produce strong contractions in muscles. These conditions produce relatively low levels of activity in mechanoreceptive afferents, and little or no activation in nociceptive afferents, so are unlikely to provoke reflexive activity [1].

\section{Conclusions}

BAS aims to prevent occurrences and recurrences of PUs in paralyzed patients by separately building up gluteal soft-tissue volume and blood circulation, and intermittently unloading the tissues under the ischium when seated. This pilot study provides proofof-concept for the ability to: 1) stimulate the HS and GM selectively with BIONs; and 2) achieve desired concomitant weight-shifting, muscle hypertrophy and perfusion benefits. It also highlights additional technological requirements including the need for a battery powered BION system, and for a mechanical pressure mapping system. Clinical refinements described include methods for securing the GM BION to the deep GM fascia, and for actively excluding high amplitude cross-simulation intraoperatively.

BION NMES may prove to be a positive therapeutic modality for PUP. This would lay the foundation for its broader application in the general PU-risk population, where two BIONs could be inserted prophylactically on each side, by percutaneous injection, to be controlled and powered autonomously via an in-chair antenna.

\section{Financial disclosure and products}

This research was funded by the Alfred Mann Institute (AMI) at the University of Southern California, which manufactured the BION devices and sponsored the study under an FDA approved Investigational Device Exemption (IDE \# G040232). Some of the authors are inventors on patents issued and pending that are assigned to AMI. None of the authors have any other financial interests or associations that may pose a conflict of interest.

Public Trials Registry

Trial Registry:

ClinicalTrials.gov

\section{Registration Identification No.:}

NCT00628602

Registry URL:

www.clinicaltrials.gov

\section{References}

[1] L.L. Baker, C.L. Wederich, D.R. McNeal, C. Newsam and R.L. Waters, Neuromuscular electrical stimulation: a practical guide 4th ed. CA Los Amigos Research and Education Institute Downey, 2000, 91-97.

[2] K.M. Bogie, X. Wang and R.J. Triolo, Long-term prevention of pressure ulcers in high-risk patients: A single case study of the use of gluteal neuromuscular electric stimulation, Arch Phys Med Rehabil 87 (2006), 585-591.

[3] H.M. Kaplan, L.L. Baker, R. Davoodi, N.T. Wong and G.E. Loeb, Preventing ischial pressure ulcers: II. Biomechanics Companion Paper, 2011.

[4] H.M. Kaplan and G.E. Loeb, Preventing ischial pressure ulcers: I. Review of neuromuscular electrical stimulation Companion Paper, 2011.

[5] H.M. Kaplan, Neuromuscular anatomical and technological considerations (Chpt 2). In Neuromuscular electrical stimulation for pressure ulcer prevention, doctoral thesis. Dept of Biomedical Engineering, University of Southern California, Los Angeles, CA, 2008, pp. 60-117.

[6] H.M. Kaplan, Pressure ulcer prevention by muscle activation II: soft tissue biomechanics (Chpt 4). In Neuromuscular electrical stimulation for pressure ulcer prevention, Doctoral Thesis Dept of Biomedical Engineering, University of Southern California Los Angeles, CA 2008, pp. 162-197.

[7] A. Lima, J. Bakker, Noninvasive monitoring of peripheral perfusion, Intensive Care Med 31 (2005), 1316-1326.

[8] National Spinal Cord Injury Statistical Center, Annual Report for the model spinal cord injury care systems. Birmingham, AL: University of Alabama, 2006, pp. 119-121.

[9] D. Popovic, LL. Baker and G.E. Loeb, Recruitment and comfort of BION implanted electrical stimulation: implications for FES applications, IEEE Trans Neural Syst Rehabil Eng 15(4) (2007), 577-586. 
[10] W. Sae-Sia, D.D. Wipke-Tevis and D.A. Williams, Elevated sacral skin temperature (Ts): A risk factor for pressure ulcer development in hospitalized neurologically impaired Thai patients, Appl Nurs Res 18(1) (2005), 29-35.

[11] M.E. Siegel, CA. Stewart, Peripheral vascular disease; Sect 1: Arterial disease and ulcers (Chpt 38), H.N. Wagner Jr, Z. Szabo and J.W. Buchanan (eds), Principles of Nuclear Medicine, 2nd Ed WB Saunders Philadelphia, PA, 1995, pp. 859-871.

[12] L.R. Solis, D.P. Hallihan, R.R.E. Uwiera, R.B. Thompson, E.D. Pehowich and V.K. Mushahwar, Prevention of pressure-induced deep tissue injury using intermittent electrical stimulation, J Appl Physiol 102 (2007), 1992-2001.
[13] S. Sprigle, W. Dunlop and L. Press, Reliability of bench tests of interface pressure, Assist Technol 15(1) (2003), 49-57.

[14] I. Swain, The measurement of interface pressure. In D. Bader, C. Bouten, D. Colin and C. Oomens (eds), Pressure Ulcer Research: Current and Future Perspectives, Springer-Verlag, Berlin, Germany, 2005, p. 56.

[15] T.K. Whitehurst, J.H. Schulman, K.N. Jaax and R. Carbunaru, The Bion ${ }^{\circledR}$ microstimulator and its clinical applications. In D.D. Zhou, E. Greenbaum (eds), Implantable Neural Prostheses 1 Biological and Medical Physics Biomedical Engineering, New York: Springer, 2009, pp. 253-273. 

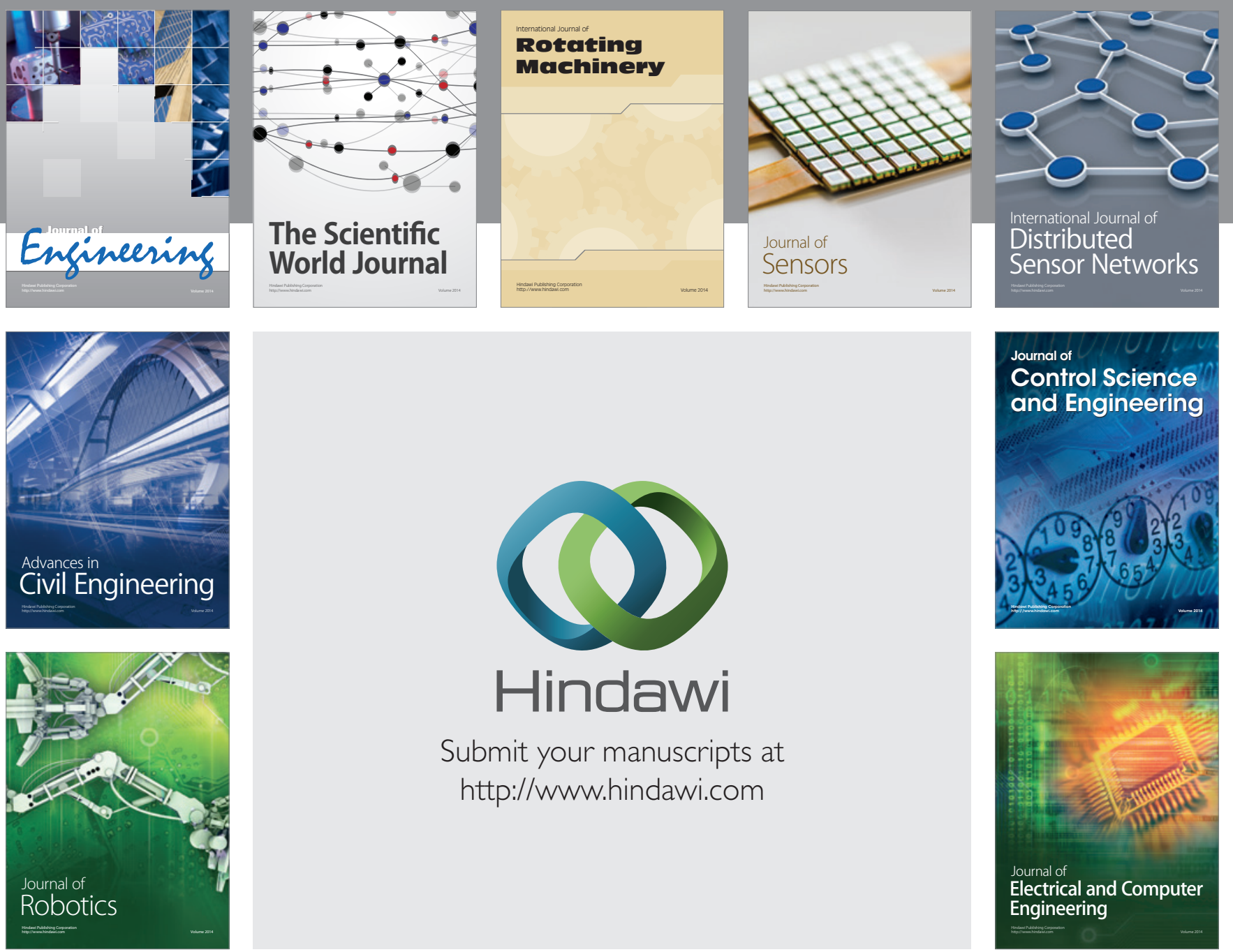

Submit your manuscripts at

http://www.hindawi.com
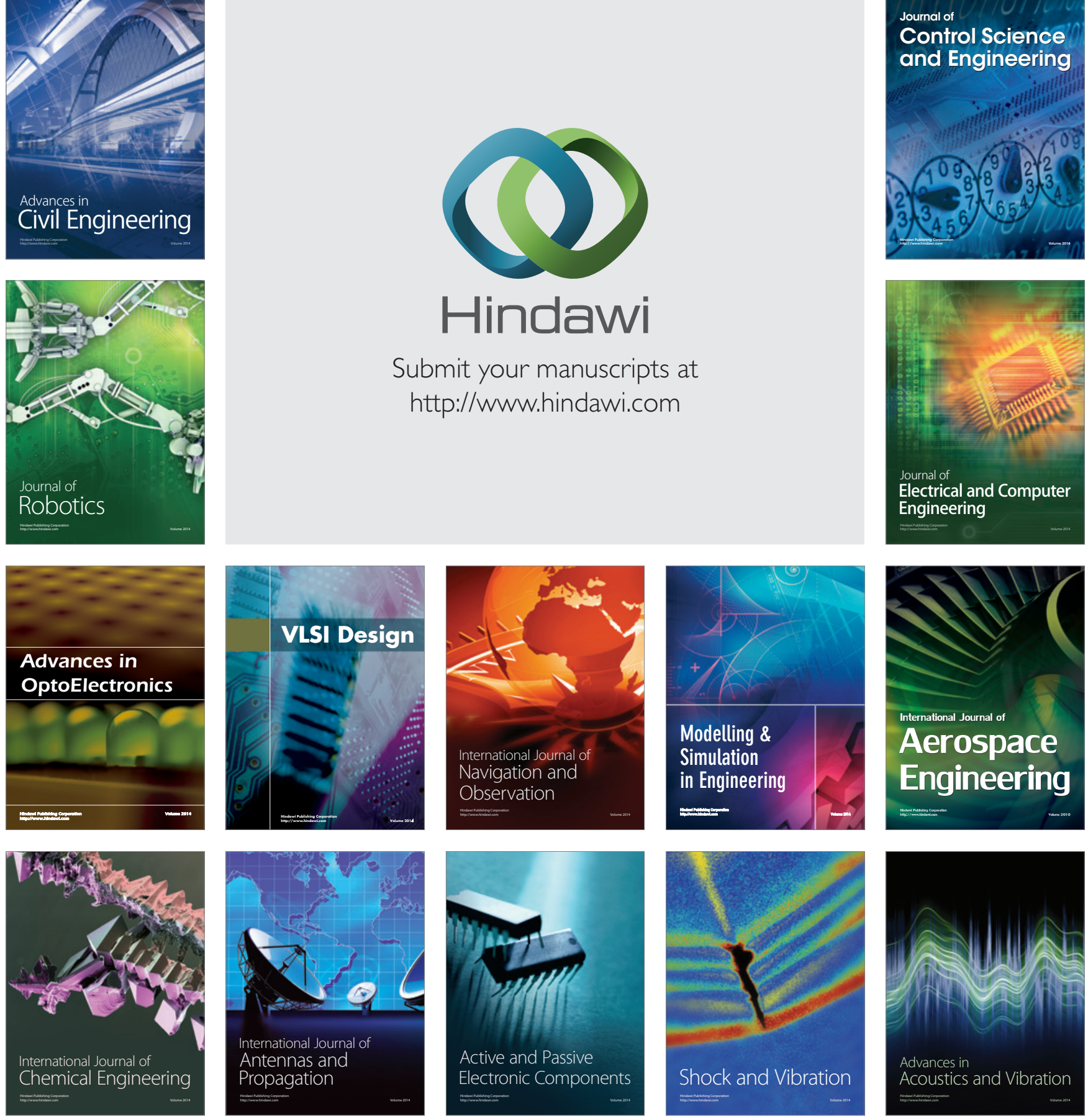